\title{
STRUCTURAL TRANSFORMATIONS IN METALLIC GLASSES
}

\author{
Marcel MIGLIERINI \\ Institute of Nuclear and Physical Engineering, Faculty of Electrical Engineering and Information Technology \\ Slovak University of Technology, Ilkovičova 3, 81219 Bratislava, Slovak Republic, tel. +421 260291 167, \\ e-mail: marcel.miglierini@stuba.sk
}

\begin{abstract}
Structural transformations that are taking place in metallic glasses have severe impact upon their performance in variety of practical applications. Their originally amorphous structure changes under elevated temperature. Eventually, partial crystallization occurs. In order to follow the development of crystallization, the investigation of heat treatment of Fe-based metallic glasses was performed. Modifications in structure were studied by employing in-situ techniques of synchrotron radiation. Namely, diffraction experiments and nuclear forward scattering of synchrotron radiation are reported. Both methods of study were performed on-fly providing information about the state of the samples in real time.
\end{abstract}

Keywords: synchrotron radiation, X-ray diffraction, nuclear forward scattering, metallic glasses, structural transformations

\section{INTRODUCTION}

The atomic arrangement in metallic glasses (MG) is not well understood at present unlike the well-defined long-range order in crystalline materials. Disordered nature of their structural arrangement gives rise to advantageous properties that initiate variety of practical applications. Nevertheless, sustainable performance of MGs suffers from two external parameters: temperature and time of operational conditions.

The transformation of structure via crystallization is one of the mechanisms that considerably affect the macroscopic physical properties of these materials. In order to understand and further optimize these properties it is inevitable to understand the microstructure and the processes that are taking place during the crystallization. The behaviour of MGs is usually investigated under static external conditions in relatively long time windows over several tens of minutes. On the other hand, the information derived by rapid data acquisition techniques is averaged over the entire volume of the sample and is limited only to one specific feature such as structural order or magnetic behaviour.

Thus, the structural characterization of MGs at the atomic level represents an ambitious mission requiring advanced experimental techniques such as those using superior intense X-rays produced by the latest generation of the synchrotron radiation sources. Yavari et al. [1] presented that the structural relaxation processes of MGs can be studied in-situ by X-ray diffraction using intensive synchrotron radiation. They showed that microscopic structural changes can be successfully correlated with macroscopic characteristics such as a thermal expansion coefficient. Furthermore, Poulsen et al. [2] demonstrated that deformation tensor components can be extracted not only in the case of crystalline materials but also from MGs using high quality synchrotron diffraction data. This concept is based on monitoring the polar angular dependence of the first maximum of a diffraction profile for different applied tensile stresses.

Bednarcik et al. [3] have studied temperature evolution of as-quenched MG ribbons during constant-rate heating by in-situ X-ray diffraction in transmission mode using high energy photons $(88 \mathrm{keV})$. A thermal expansion of the amorphous alloys has provided an insight into the thermally activated effects such as relaxation and crystallization via analysis of a series of XRD patterns in reciprocal space.

Köster et al. [4] investigated formation of ultrafine microstructure of a FINEMET-type alloy by in-situ time resolved diffraction of synchrotron radiation (DSR). The size of the primary crystals increased with a square of annealing time. A role of diffusion of impurities upon the transformation was also emphasised.

Zr-based bulk MGs with and without Be were studied by Mechler et al. [5] using DSR. Differences in glass forming abilities were pointed out to be responsible for different icosahedral arrangements in short-range order in both alloys. It was shown that chemical short-range order plays a crucial role in thermal stability of the amorphous alloys.

Kinetics of crystallization of FINEMET-type MG [6, 7] and the effect of Co upon the kinetics of crystallization [8] were also studied using in-situ DSR.

Kinetics of crystallization was recently reported in similar alloys by in-situ X-ray diffractometry using synchrotron radiation with low energy (7 keV) [9].

Another method that became feasible with the availability of the third generation synchrotron sources is nuclear resonant scattering (NRS) [10]. NRS uses atoms of ${ }^{57} \mathrm{Fe}$ as probes of the local magnetic and electronic properties in the investigated samples and provide information on hyperfine interactions. Application of this technique is helpful in revealing the mutual relation between the magnetic arrangement and the structure of the studied materials and it can be used in a dynamic in-situ regime. Several applications of this technique to different problems of condensed matter physics including NFS in glassy and nanoscale materials are reviewed in [11].

In this paper we provide examples of the use of synchrotron radiation for the investigation of structural changes that are taking place in selected MGs exposed to continuous heat treatment with rapidly increasing temperature. Structural transformations are followed by 
DSR whereas evolution of hyperfine interactions is discussed using the results of NFS.

\section{EXPERIMENTAL DETAILS}

The investigated MGs were prepared in a form of ribbons using the technique of a planar flow casting of melt on a rotating wheel. Alloys with the nominal composition of $\left(\mathrm{Fe}_{1-x} \mathrm{Co}_{x}\right)_{76} \mathrm{Mo}_{8} \mathrm{Cu}_{1} \mathrm{~B}_{15}, \quad x=0,0.25$ and $\mathrm{Fe}_{90} \mathrm{Zr}_{7} \mathrm{~B}_{3}$ were investigated.

Structural transformations were studied during continuous heating with the ramp of $10 \mathrm{~K} / \mathrm{min}$ in a vacuum. DSR was performed at the KMC-2 experimental station at BESSY, Berlin with the energy of $7 \mathrm{keV}(\lambda=$ $0.177121 \mathrm{~nm})$.

Evolution of hyperfine interactions was inspected by NFS as a function of dynamically varying temperature and with respect to time in isothermal experiments. The research was carried out at the Nuclear Resonance end station ID22N of ESRF in Grenoble. We used $14.413 \mathrm{keV}$ photons with an energy resolution of about $3 \mathrm{meV}$. The samples were placed inside a vacuum furnace. The NFS spectra were continuously recorded with an acquisition time of 1 min up to the desired temperature and/or time.

\section{RESULTS AND DISCUSSION}

\subsection{Diffraction of Synchrotron Radiation}

Diffractograms of the studied MG were obtained during continuous temperature increase by the help of a 2$\mathrm{D}$ detector. The latter covers sufficiently broad range of angles to allow the main reflection peak to be recorded without any need for additional movement. This has enabled fast collection of the diffraction data every 10 sec. (a)

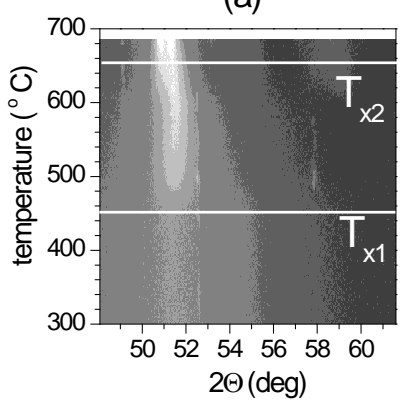

(b)



Fig. 1 Contour plots of diffractograms from $\left(\mathrm{Fe}_{1-x} \mathrm{Co}_{x}\right)_{76} \mathrm{Mo}_{8} \mathrm{Cu}_{1} \mathrm{~B}_{15}$ alloy for $x=0$ (a) and $x=0.25$ (b). Crystallization temperatures $\mathrm{T}_{\mathrm{x} 1}$ and $\mathrm{T}_{\mathrm{x} 2}$ are indicated by horizontal lines

The obtained diffractograms were stacked according to the temperature of measurement. They are presented in Fig. 1 in a form of contour plots. At low enough temperature, the investigated MGs are fully amorphous and characterized by broad peaks positioned at about $51.5^{\circ}$. With increasing temperature, the first crystallization starts at $T_{x 1}$ where narrow reflections that correspond to an emerging crystalline phase appear. They were identified to be bcc-Fe and bcc-Fe,Co for $x=0$ and 0.25 , respectively

Further enhancement of temperature to $\mathrm{T}_{\mathrm{x} 2}$ causes the second crystallization step to occur at which additional crystalline phases are created.

Quantitative analysis of the crystallization kinetics was performed by decomposition of the main diffraction peak into a broad line assigned to the residual amorphous phase and a narrow one of the bcc nanograins. As seen from Fig. 2 , where the crystalline fraction is plotted against the temperature, presence of Co in the master alloy decreases the onset of crystallization $T_{x 1}$ by about $57^{\circ}$.

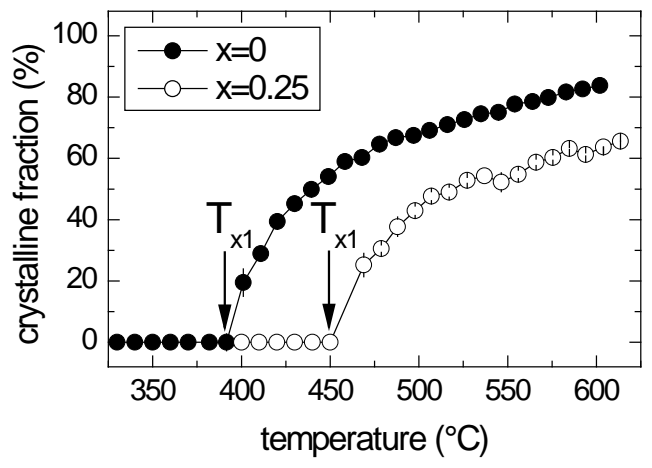

Fig. 2 Crystalline fraction as a function of temperature

The lattice parameter of the crystalline phase can be derived from the position of the corresponding narrow reflection. The results presented in Fig. 3 demonstrate an initial decrease in the lattice parameters with temperature for both alloys. This is caused by formation of the respective crystalline phases whose crystalline lattices evolves and acquire their optimal structural arrangements. A rapid increase in the lattice parameters at high temperatures is associated with a thermal expansion of the lattices. Substitution of Co decreases the lattice parameter.



Fig. 3 Lattice parameter as a function of temperature

The lattice parameter for $x=0$ is quite high in comparison with $0.2866 \mathrm{~nm}$ for pure bcc-Fe lattice at room temperature. This is most probably due to incorporation of some Mo atoms into the Fe nanocrystals.

As we have demonstrated above, DSR can be effectively used to describe the structural properties of MGs that are undergoing temperature induced transformations including first and second crystallization steps. In order to look at the magnetic order of the resonant atoms, NFS is the only possibility if we would like to study these features in real time during in-situ heat treatment. 


\subsection{Nuclear Forward Scattering of Synchrotron Radiation}

Formation of nanograins of a newly developed crystalline phase is indirectly revealed via the corresponding hyperfine interactions. They even enable identification of the residual amorphous matrix as well as the so-called interface regions. The latter represent those iron atoms that are located at the surfaces of the nanograins. Using isothermal experiments, the kinetics of crystallization and close description of time evolution of the hyperfine interactions in all structurally different regions was studied.

The time NFS spectra were collected during isothermal heat treatment at a temperature which was chosen to be close to the onset of crystallization of the investigated $\mathrm{Fe}_{90} \mathrm{Zr}_{7} \mathrm{~B}_{3}$ NANOPERM alloy. The spectra have reveal rapid development that takes place shortly after the beginning of the annealing process as seen from the contour plot in Fig. 4a. This is a direct consequence of the changes in the hyperfine interactions due to modifications of the nearest-neighbourhood of the iron atoms connected with the emerging ferromagnetic nanocrystalline grains. Examples of some selected NFS spectra are shown in Fig. 4b together with the theoretically calculated fitting curves.

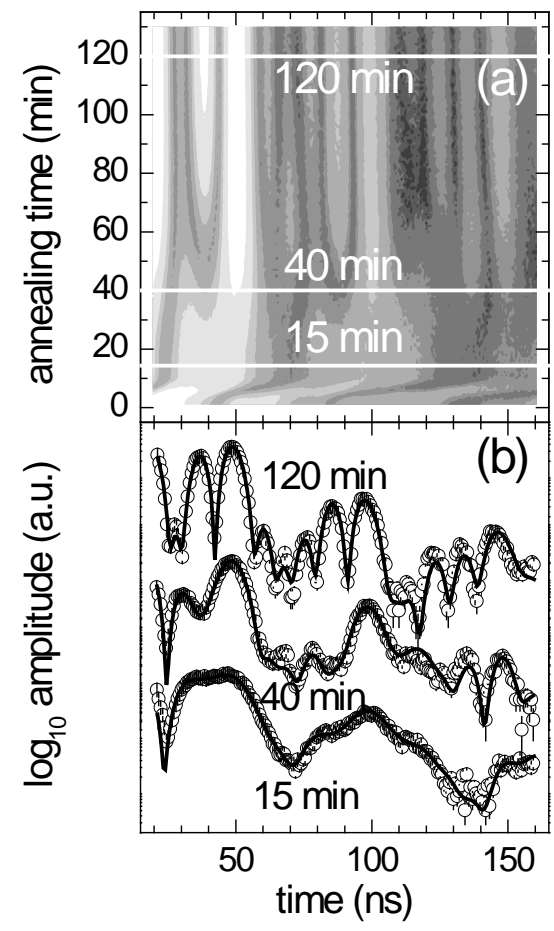

Fig. 4 Contour plot (a) obtained from isothermal NFS experiment upon $\mathrm{Fe}_{90} \mathrm{Zr}_{7} \mathrm{~B}_{3} \mathrm{MG}$ and several selected records (b) obtained after the indicated times of annealing. Solid lines are fits to the experimental data (open symbols)

By performing quantitative analysis of the NFS spectra we were able to disclose the nucleation and growth of the iron nanograins to fine details by accessing separately atoms located in their interior (bulk), at their surfaces as well as those that belong to the residual amorphous matrix. Evolution of the hyperfine magnetic fields of the bulk and surface regions of the nanocrystallites are plotted as a function of the annealing time in Fig. 5a. The developed crystallization model (solid lines in Fig. 5b) allowed for complete understanding and explanation of the experimental data (open symbols in Fig. 5b).

The newly born nanocrystals are stabilized once their structure is well developed. This is demonstrated by stabilization of the magnetic hyperfine fields after about 45 min in Fig. 5a. During this grain growth process, the relative contribution of the surface atoms dominates that of the bulk atoms, which appears only later and prevails after about $70 \mathrm{~min}$ of annealing (Fig. 5b).

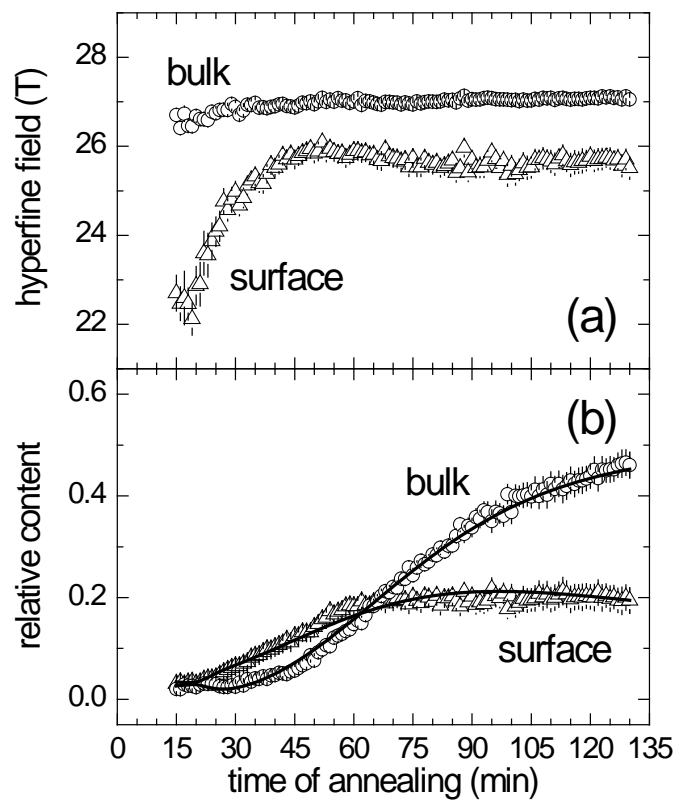

Fig. 5 Magnetic hyperfine fields (a) and the relative contents (b) of the bulk and surface iron atoms plotted against the time of annealing at $470{ }^{\circ} \mathrm{C}$

The solid lines in Fig. 5b are fits to the experimental data (open symbols) according to a model of crystallization which we have also developed for this purpose and takes into consideration contributions both from the bulk as well as surface atoms.

\section{CONCLUSIONS}

In order to ensure an effective use of metallic glasses in practical application, their response to the external conditions should be known. In particular, because elevated temperature during the operation can trigger out crystallization that consequently significantly affects their macroscopic properties and performance, we have followed the effect of temperature by in-situ experiments using synchrotron radiation.

It was shown that diffraction of synchrotron radiation provides valuable information about structural transformations that are taking place in metallic glasses exposed to annealing. The relative amount of newly formed crystalline phases as well as their characteristics including the lattice parameters can be obtained from the analysis of the diffractograms recorded during short time intervals.

In order to assess the magnetic states of the investigated MGs, the method of nuclear forward scattering of synchrotron radiation was applied. This technique enables separate evaluation of the contributions 
that stem from structurally different regions within the investigated samples including the newly formed nanocrystals, the residual amorphous matrix as well as interface (surface) regions.

Especially important is the fact that by using NFS it is now possible to experimentally identify the so-called interface regions formed by the atoms located at the surfaces of the nanograins. The interface component in the NFS spectra prevails at the stages of early crystallization when the majority of atoms are located at the nanograin surface. At advanced crystallization stages the bulk fraction, resulting from the growth of the nanograins size as well as of their number, increases.

Employing in-situ nuclear forward scattering during isothermal crystallization, the process of nanograin formation was investigated in details. Consequently, the birth and development of the nanosized crystalline grains embedded in amorphous precursor was followed in real time revealing how the nanograins are formed especially at the very early stages of isothermal annealing. Even though the overall formation of nanocrystals can be deduced also from other analytical techniques, the approach proposed here provides the possibility to study independently iron atoms positioned at different structural sites within the investigated alloy owning to their sitespecific hyperfine interactions.

Owing to its extremely high brilliance, the synchrotron radiation ensures experiments in time scales that are not accessible using conventional laboratory sources of radiation. In this way, completely new experiments can be performed that will help with the elucidation of technologically important details on the behaviour of metallic glasses.

\section{ACKNOWLEDGMENT}

This publication is the result of the project implementation Centre of Competence in New Materials, Advanced Technologies and Energy, ITMS 26240220073 supported by the Research \& Development Operational Programme funded by the ERDF.

\section{REFERENCES}

[1] YAVARI, A. R. - Le MOULEC, A. - INOUE, A. NISHIYAMA, N. - LUPU, N. - MATSUBARA, E. - BOTTA, W. J. - VAUG-HAN, G. - Di MICHIEL, M. - KVICK, Å.: Excess free volume in metallic glasses measured by X-ray diffraction, Acta Materialia, vol. 53, pp. 1611-1619, 2005.

[2] POULSEN, H. F. - WERT, J. A. - NEUEFEIND, J. - HONKIMAKI, V. - DAYMOND, M.: Measuring strain distribution in amorphous materials, Nat. Mater., vol. 4, pp. 33-36, 2005.

[3] BEDNARCIK, J. - MIGLIERINI, M. - CURFS, C. - FRANZ, H.: Thermal expansion of NANOPERMtype alloys from in-situ X-ray diffraction, AIP Conf. Proc., Vol 1258, (eds. J. Tuček and M. Miglierini), AIP, Melville, New York, pp. 1-8, 2010.
[4] KÖSTER, U. - SCHÜNEMANN, U. - BLANKBEWERSDORFF, M. - BRAUER, S. - SUTTON, M. - STEPHENSON, G. B.: Nanocrystalline materials by crystallization of metal-metalloid glasses, Mater. Sci. Eng. A, vol. 133, pp. 611-615, 1991.

[5] MECHLER, S. - SCHUMACHER, G. - ZIZAK, I. MACHT, M. P. - WANDERKA, N.: Correlation between icosahedral short range order, glass forming ability, and thermal stability of $\mathrm{Zr}-\mathrm{Ti}-\mathrm{Ni}-\mathrm{Cu}-(\mathrm{B})$ glasses, Appl. Phys. Lett., vol. 91, art. n. 021907, 2007.

[6] BORREGO, J. M. - CONDE, C. F. - MILLÁN, M. - CONDE, A. - CAPITÁN, M. J. - JOULAUD, J. L.: Nanocrystallization in $\mathrm{Fe}_{73.5} \mathrm{Si}_{13.5} \mathrm{~B}_{9} \mathrm{Cu}_{1} \mathrm{Nb}_{1} \mathrm{X}_{2}$ $(\mathrm{X}=\mathrm{Nb}$, Mo and V) Alloys Studied by X-ray Synchrotron Radiation, Nanostructured materials, vol. 10, pp. 575-583, 1998.

[7] AFONSO, C. R. M. - BOLFARINI, C. - BOTTA FILHO, W. J. - KIMINAMIM, C. S.: In-situ crystallization of amorphous $\mathrm{Fe}_{73-\mathrm{x}} \mathrm{Nb}_{\mathrm{x}} \mathrm{Al}_{4} \mathrm{Si}_{3} \mathrm{~B}_{20}$ alloys through synchrotron radiation, J. NonCrystalline Solids, vol. 352, pp. 3404-3409, 2006.

[8] BEDNARČÍK, J. - NICULA, R. - STIR, M. BURKEL, E.: In situ X-ray diffraction investigation of nanocrystallization of amorphous $\mathrm{Co}-\mathrm{Fe}-\mathrm{Zr}-\mathrm{B}$ alloys, J. Magn. Magn. Mat., vol. 316, pp. 823-826, 2007.

[9] MIGLIERINI, M. - KAŇUCH, T. - PAVÚK, M. ŠVEC, P. - SCHUMACHER, G. - ZIZAK, I. JIRÁSKOVÁ, Y.: Structural Transformations in NANOPERM-Type Alloys Studied by Mössbauer Spectrometry and Diffraction of Synchrotron Radiation, Hyperfine Int., vol. 183, pp. 31-35, 2008.

[10] SMIRNOV, G. V.: General properties of nuclear resonant scattering, Hyperfine Int., vol. 123/124, pp. 31-77, 1999.

[11] RÜFFER, R.: Nuclear resonance scattering, C.R. Physique, vol. 9, pp. 595-607, 2008.

Received November 19, 2012, accepted March 3, 2013

\section{BIOGRAPHY}

Marcel Miglierini was born in 1956 in Bratislava. He graduated from the Slovak University of Technology (STU) in Bratislava in 1981. In 1995 he has received the DrSc degree. Since 1983 he works at the Institute of Nuclear and Physical Engineering, Faculty of Electrical Engineering and Information Technology, STU, since 1997 as a full professor in condensed matter physics. His research activities comprise study of disordered systems by non-destructive spectroscopy methods. He is a member of the Steering Committee of the Consortium CENTRALSYNC which ensures access to the ESRF for Slovak scientists. 